\title{
SOCIOAVA_EP: UM OBJETO DE APRENDIZAGEM SOBRE ESTRATÉGIAS PEDAGÓGICAS COM FOCO NAS INTERAÇÕES SOCIAIS EM AMBIENTES VIRTUAIS DE APRENDIZAGEM
}

\section{Ana Carolina Ribeiro Ribeiro - PPGEDU/UFRGS - carolribeiro2@ gmail.com} Patricia Alejandra Behar - PPGIE/PPGEDU/UFRGS - pbehar@terra.com.br

Resumo: Esse artigo apresenta o planejamento, a implementação e a avaliação de Objeto de Aprendizagem (OA) SocioAVA_EP - Estratégias Pedagógicas a partir das interações sociais em Ambientes Virtuais de Aprendizagem. A avaliação do material foi realizada em um curso de extensão que contou com a participação de 30 professores atuantes da EAD, em que foram discutidas questões relacionadas aos desafios da modalidade e as possibilidades dos Ambientes Virtuais de Aprendizagem disponíveis hoje. A análise dos dados demonstrou, entre outras questões, a importância de materiais que possibilitem a aproximação e a atenção de docentes a esses aspectos, bem como, a realização de atividades que permitam com que os mesmos reflitam sobre os desafios encontrados pelos estudantes.

Palavras-chave: estratégias pedagógicas; interações sociais; objeto de aprendizagem; ambientes virtuais de aprendizagem

\section{SOCIOAVA_EP: A LEARNING OBJECT ABOUT PEDAGOGICAL STRATEGIES FOCUSING ON SOCIAL INTERACTIONS IN VIRTUAL LEARNING ENVIRONMENTS}

\begin{abstract}
This article presents the planning, implementation and evaluation of Learning Object (OA) SocioAVA_EP - Pedagogical Strategies from social interactions in Virtual Learning Environments. The evaluation of the material was carried out in an extension course that was attended by 30 active teachers of EAD, in which issues related to the challenges of the modality and the possibilities of the Virtual Learning Environments available today were discussed. The analysis of the data showed, among other issues, the importance of materials that allow the approach and attention of teachers to these aspects, as well as the performance of activities that allow them to reflect on the challenges encountered by students.
\end{abstract}

Keywords: pedagogical strategies; social interactions; learning object; virtual learning environments

\section{INTRODUÇÃO}

O objetivo deste artigo é apresentar o planejamento, desenvolvimento e implementação do objeto de aprendizagem SocioAVA_EP - Estratégias Pedagógicas a partir das interações sociais em Ambientes Virtuais de Aprendizagem. Este tem o objetivo de discutir o desenvolvimento de estratégias pedagógicas a partir do mapeamento das interações sociais em um Ambiente Virtual de Aprendizagem (AVA).

Entende-se, a partir de uma perspectiva piagetiana, que a construção do indivíduo ocorre durante sua interação no/com o meio; isto é, a interação do indivíduo com o objeto de conhecimento e dele com outros indivíduos. Ao se transpor essas interações para o meio digital, através das ferramentas disponíveis em AVA, uma série de estratégias pedagógicas podem ser desenvolvidas. 
As estratégias pedagógicas são compreendidas nesta abordagem como um conjunto de ações educacionais, colocadas em prática pelo professor, que visam alcançar os objetivos que levam à construção do conhecimento. Nesse contexto, com base nos aspectos sociais, elas podem abranger a utilização de novas ferramentas, novas aplicações para ferramentas já utilizadas e de dinâmicas que busquem uma maior integração entre os participantes de um curso ou disciplina. Estes poderão ser professores, alunos e/ou monitores/tutores.

Com base nessas questões, o Objeto de Aprendizagem (OA) SocioAVA_EP tem por função auxiliar o docente na condução de discussões sobre estratégias pedagógicas orientadas às formas de interação social em AVA. Desse modo, entende-se que contribui com a formação inicial e continuada de professores que atuam, principalmente, na EAD, promovendo subsídios para a prática pedagógica realizada em um ambiente virtual.

Para abordar a importância do tema principal do objeto, na próxima sessão serão discutidas as interações sociais em ambientes virtuais de aprendizagem, seguido da relevância de se pensar estratégias pedagógicas específicas para estes aspectos na EAD. Para compreender as etapas de desenvolvimento do SocioAVA na metodologia serão apresentadas as etapas de desenvolvimento do mesmo, além dos processos realizados para sua avaliação. Na quinta seção são apresentados os resultados de aplicação e trechos de falas dos participantes envolvidos. Por fim, na sétima seção, são apresentadas as considerações que indicam a relevância do objeto em cursos de formação bem como as possibilidades de uso do OA pelos professores.

\section{INTERAÇÕES SOCIAIS E ESTRATÉgIAS PEDAGÓgICAS NA EDUCAÇÃO A DISTÂNNCIA}

Entende-se que na Educação a Distância é de extrema importância a valorização do papel do estudante na aprendizagem, visto que, por diversos momentos, encontra-se distante fisicamente do professor e dos colegas. Para transcender essa distância, as tecnologias digitais têm tido um papel fundamental, levando inovação e abrindo novos caminhos no campo educacional. Essas tecnologias possibilitam o ensino e a aprendizagem fora dos prédios institucionais, mas, mais do que isso, permitem formas diferenciadas e interessantes de ensinar e aprender. A interação constante entre os atores da EAD é fundamental para o êxito pedagógico nesta modalidade.

Destaca-se, no entanto, que para que essas interações ocorram é fundamental propor atividades que visem as trocas entre os sujeitos, sendo necessário repensar a ação educacional, através da valorização de uma formação mais integral, não se atendo apenas ao aspecto cognitivo. Piaget $(1973 ; 2014)$ afirma que o desenvolvimento intelectual bem estruturado tem como pressuposto a devida atenção à afetividade e às interações sociais. Em outros termos, os processos cognitivos são assumidos como profundamente interligados às dimensões afetivas e sociais.

Para Piaget (1973) o conhecimento humano é essencialmente coletivo e é a vida social que constituirá um dos fatores mais essenciais da formação e do crescimento dos conhecimentos pré-científicos e científicos. Em termos de desenvolvimento, o autor destaca que, enquanto o desenvolvimento orgânico individual depende de transmissões hereditárias, o desenvolvimento mental é em parte condicionado pelas transmissões sociais ou educativas. Essas transmissões interferem nesse processo antes mesmo da linguagem, através de aspectos como a imitação, sem ocorrência, no entanto, de modificação da inteligência pré-verbal. É a partir da aquisição da linguagem que as interações sociais apresentam, cada vez mais, um papel importante nesse processo, promovendo mudanças de pensamento. Há, dessa forma, uma interdependência 


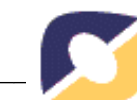

crescente, a partir da construção progressiva das operações intelectuais, entre as interações interindividuais e os fatores mentais. Uma vez constituídas as operações, há o estabelecimento de um equilíbrio entre o mental e o social e o sujeito, enquanto membro adulto da sociedade, não poderia mais pensar fora desta socialização acabada (PIAGET, 1973).

Entre as principais tecnologias que dão suporte ao desenvolvimento da Educação a Distância, atualmente, podem ser destacados os Ambientes Virtuais de Aprendizagem (AVA). Borba e Gasparini (2015, p. 2) destacam que "o uso desses ambientes vem aumentando consideravelmente nos últimos anos e os AVAs geram diariamente uma quantidade enorme de dados sobre os alunos". Esses evidenciam o acesso, as formas de interação, o tempo de conexão dos mesmos, entre outras questões. Dessa forma, ao acompanhar esses dados, o professor tem a oportunidade de construir, adequar e direcionar sua prática pedagógica de forma a torná-la mais próxima das necessidades de seus alunos.

Os sujeitos que interagem através de um AVA vivenciam experiências sociais e afetivas durante as trocas realizadas. $\mathrm{O}$ sentido de pertencer a um grupo, o entusiasmo, o interesse e a satisfação da tarefa cumprida podem favorecer, dessa forma, a construção do conhecimento e a busca por novas aprendizagens. Essas iniciativas dependem, no entanto, das estratégias pedagógicas empregadas pelo professor no ambiente utilizado.

Souza, Sartori e Roesler (2008) salientam que, como a mediação do processo educativo a distância geralmente ocorre através de um Ambiente Virtual de Aprendizagem, dispõe-se de uma série de ferramentas que possibilitam a comunicação síncrona e assíncrona entre os sujeitos. Permite-se, assim, a implementação de estratégias pedagógicas que promovam o diálogo e a participação dos alunos. Dessa forma, além de promover a construção de competências relacionadas à comunicação por todos os atores, promovem a criação de espaços de interação e de construção coletiva através de possibilidades concretas de trabalho colaborativo. Pensar esses aspectos se torna importante, pois cada ferramenta exige uma série de habilidades diferenciadas "e propiciam diferentes estratégias pedagógicas, que exigem participação em tempo real ou diferida, possibilitando a expressão, a intervenção e a colaboração para a construção coletiva do conhecimento" (SOUZA, SARTORI E ROESLER, 2008, p.331).

Com base nestas questões, compreender-se que é fundamental considerar estratégias pedagógicas que atentem para as interações sociais no ambiente virtual de aprendizagem utilizado em um curso a distância. Neste sentido, conforme explicitado anteriormente, foi desenvolvido um objeto de aprendizagem voltado para a formação de professores que consiste em problematizar a importância destas estratégias no processo educativo.

\section{OBJETO DE APRENDIZAGEM SOCIOAVA_EP}

No contexto educacional, vê-se uma crescente necessidade de inclusão das tecnologias digitais, bem como a discussão sobre as relações sociais nos ambientes virtuais de aprendizagem. Paralelamente, a produção de materiais educacionais digitais (MED) tem crescido, principalmente, nos meios acadêmicos, como uma possibilidade para a apresentação de conteúdos e materiais de uma forma mais interativa e lúdica. $\mathrm{O}$ termo objeto de aprendizagem é utilizado neste artigo como sinônimo de MED já que as características são semelhantes.

De acordo com Nesi et al (2019, p. 559) "estudos sobre OA estão sendo realizados há algum tempo, mas ainda não se chegou a uma definição universalmente aceita". Behar et al (2009) considera um Objeto de Aprendizagem, qualquer material 
digital (vídeos, sons, páginas HTML, animações, etc) que possua fins educativos com embasamento pedagógico. Dessa forma, "são destinados a situações de aprendizagem tanto na modalidade à distância quanto semipresencialmente ou presencialmente" (2009, p.67). Assim, nesse estudo, compreende-se Objeto de Aprendizagem como qualquer material/recurso digital que seja formado por textos, vídeos, imagens, mapas, apresentações, páginas Web, entre outras. Os OA's podem ser utilizados como módulos de um determinado conteúdo ou possuem uma estrutura completa, sendo mais abrangente.

Para o desenvolvimento do OA contou-se com a contribuição de educadores e webdesigners de um Núcleo de Pesquisa de uma Universidade pública do sul do Brasil. Como metodologia, foi utilizada a ConstruMED (TORREZZAN, 2014), que consiste em uma metodologia para a construção de Materiais Educacionais Digitais (MED) baseados no Design Pedagógico. De acordo com Torrezzan e Behar (2013), o Design Pedagógico é uma concepção teórica que visa "orientar a aplicação de recursos digitais em materiais educacionais por meio do concomitante planejamento técnico, gráfico e pedagógico". Cabe destacar que, dessa forma, que nessa pesquisa entende-se Objeto de Aprendizagem como sinônimo de material educacional digital.

A construção do objeto seguiu as cinco fases propostas pela metodologia ConstruMED (TORREZZAN, 2014):

Fase 1 - Preparação: fase de definição da equipe e das características básicas do tema/usuários. Abrange a organização da equipe de trabalho, a definição do tema abordado pelo MED/OA, a caracterização do público-alvo e a elaboração dos objetivos pedagógicos.

Fase 2 - Planejamento: período de elaboração do conteúdo e delimitação do mesmo; o planejamento das atividades envolvendo a aplicação prática da teoria estudada; o levantamento de materiais de apoio; a preparação do roteiro do OA abrangendo título do objeto, objetivo e resumo geral do conteúdo; a definição da estrutura, a partir do conteúdo de cada página e das diferentes mídias que serão incluídas; a definição e a elaboração dos recursos pedagógicos e tecnológicos; o planejamento da identidade visual das interfaces gráficas; o levantamento de critérios de usabilidade e acessibilidade; a elaboração do mapa de navegação do OA; e, por fim, a seleção de softwares e códigos de programação informática passíveis de serem utilizados para implementar o OA.

Fase 3 - Implementação: fase de construção digital do Objeto de Aprendizagem, que abrange o desenvolvimento propriamente dito, incluindo a elaboração dos primeiros protótipos dos objetos até alcançar a sua versão final (após a fase 4 - avaliação). Implica a análise e revisão do conteúdo elaborado e disposto em cada módulo e interface do OA; a organização do texto de apresentação disponibilizado na página inicial e das suas páginas auxiliares: Material de Apoio, Glossário, Créditos etc; idealização do wireframe das interfaces gráficas, ou seja, setorização, em cada interface, dos elementos que ela contém (logo, textos, botões de navegação, imagens, etc.); implementação do OA a partir do roteiro, conteúdo pedagógico, mapa de navegação e wireframe.

Fase 4 - Avaliação: fase de análise do funcionamento do OA e da adequação aos objetivos técnicos, gráficos e pedagógicos. A partir dessa análise são realizados ajustes finais que incluem a testagem da utilização do MED/OA a partir da visão do usuário/aluno, anotações sobre a necessidade de reparos e aprimoramentos; testes de funcionamento de links, botões e animações; análise dos materiais de acordo com os objetivos pedagógicos e do design gráfico das interfaces; utilização do OA em diferentes navegadores.

Fase 5 - Distribuição: fase de armazenamento e disponibilização do MED/OA, destinada à distribuição do OA construído e já avaliado. Para a disponibilização em sites 
e repositórios são descritos os metadados que serão solicitados durante o processo de envio ao repositório e que são destinados a caracterizar o referido OA para este ser encontrado em buscas online.

O Objeto de Aprendizagem SocioAVA_EP trata de Estratégias Pedagógicas a partir das interações sociais em Ambientes Virtuais de Aprendizagem. As estratégias pedagógicas são compreendidas nesta abordagem como um conjunto de ações educacionais, colocadas em prática pelo professor, que visam alcançar os objetivos que levam à construção do conhecimento. Por isso, a proposta do objeto tem a intenção de despertar o interesse dos professores no uso de ferramentas de interação e formas de analisar como interações ocorrem em um ambiente virtual. Os usuários poderão discutir essas (e outras) questões com o objetivo de propor estratégias pedagógicas. Assim, o OA SocioAVA_EP, ao abordar a influência das estratégias pedagógicas com base nos aspectos sociais na educação, focando na Educação a Distância, pode auxiliar no aprimoramento da prática pedagógica do professor, de modo a torná-la cada vez mais próxima das necessidades de seus alunos.

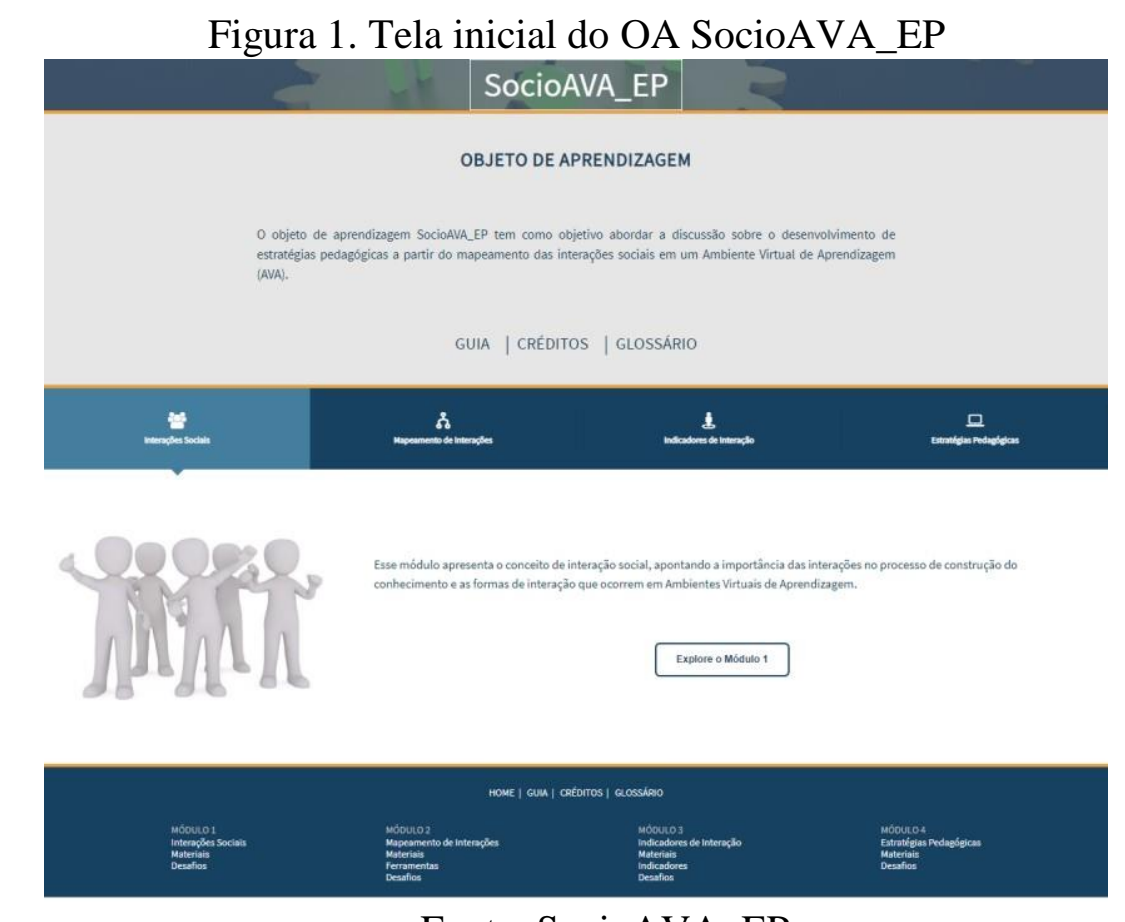

Fonte: SocioAVA_EP

Com base nessas questões, o OA SocioAVA_EP tem por função auxiliar o docente na condução de discussões sobre estratégias pedagógicas orientadas às formas de interação social em AVA. Desse modo, entende-se que contribui com a formação inicial e continuada de professores que atuam, principalmente, na EAD, promovendo subsídios para a prática pedagógica realizada em um ambiente virtual.

Assim, o OA trata dos seguintes tópicos, divididos em quatro módulos, que podem ser vinculados às discussões a serem desenvolvidas em aulas:

1. Interações sociais na Educação - explora brevemente o tema interações sociais, apontando a importância das mesmas no processo de construção do conhecimento e formas de interagir em AVA (Figura 2).

2. Mapeamento de Interações Sociais, aborda teorias e pesquisas que visam apresentar formas de mapear as interações, tais como análise de redes sociais e estudos sociométricos que podem ser realizados no AVA de aplicação; 
3. Indicadores Sociais, explicita as formas de comportamentos sociais que podem ser observados em um espaço educativo, principalmente em um AVA;

4. Estratégias Pedagógicas com base nos aspectos sociais e nas categorias abordadas nos módulos anteriores, são apresentadas algumas ações com o intuito de fazer com que professores e estudantes de licenciatura possam construir suas próprias estratégias pedagógicas que devem ser adotadas em um AVA. Através desse módulo, especificamente, busca-se o compartilhamento de estratégias pedagógicas pelos usuários do objeto e dos participantes de cursos e disciplinas em que o mesmo será utilizado. Dessa forma, foi disponibilizado um espaço de envio de propostas e experiências de modo a formar um banco de dados dinâmico. Espera-se que a formação desse banco contribua para o aprimoramento do próprio objeto, de forma a enriquecer constantemente os materiais disponibilizados.

Figura 2. Tela do Módulo Interações Sociais na Educação

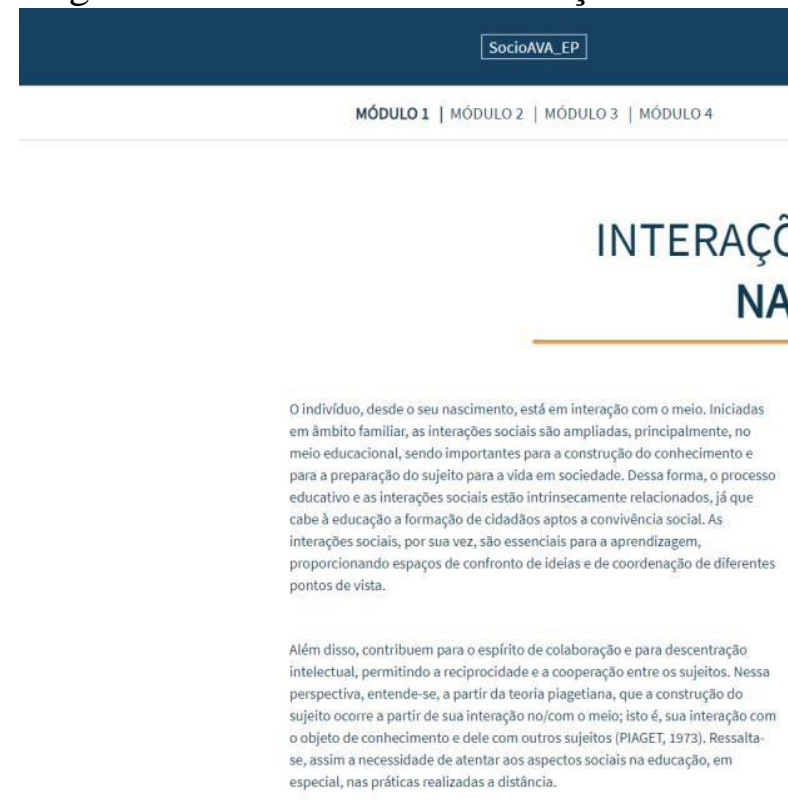

Fonte: SocioAVA_EP

Para cada módulo foram disponibilizados textos explicativos e materiais em diferentes formatos, como vídeos, imagens, animações, artigos e infográficos. Além disso, foram propostos desafios visando reflexões, trocas entre os usuários e a prática dos conteúdos abordados. São incluídas também páginas de Guia, apresentando questões referentes à aplicação do OA, Glossário (Figura 3), com as definições dos termos utilizados, e Materiais de Apoio, visando a ampliação do tema estudado e sua aplicação em diferentes contextos.

Figura 3. Tela de Glossário 


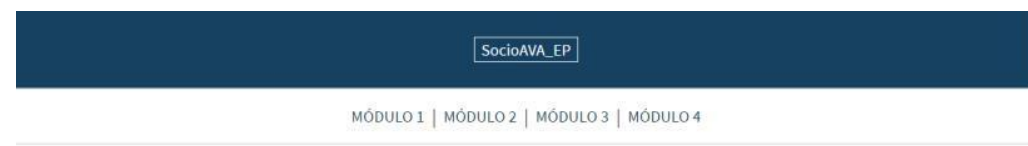

\section{GLOSSÁRIO}

CONCEITOS

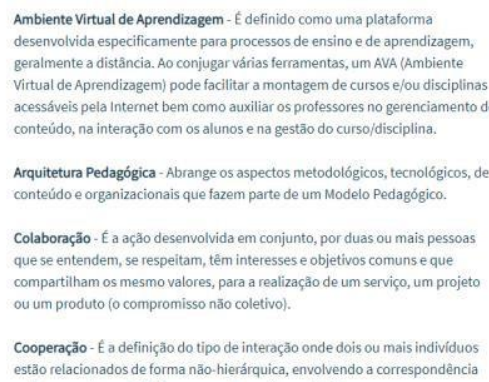

Fonte: SocioAVA_EP

A seguir, é apresentado o processo de avaliação do objeto.

\section{METODOLOGIA E RESULTADOS}

A presente pesquisa foi realizada em uma abordagem qualitativa do estudo de caso. Participaram desta pesquisa 30 professores atuantes em cursos realizados a distância em diferentes instituições ${ }^{1}$. A investigação foi realizada no segundo semestre de 2018, sendo o SocioAVA_EP utilizado como conteúdo principal e em combinação com um AVA a fim de propiciar uma maior interação (com a utilização das funcionalidades) entre os seus usuários.

Ao longo do curso foram desenvolvidos desafios incluídos no OA, sendo utilizadas para a coleta de dados as ferramentas disponíveis no ROODA. Entre elas destacam-se as de interação, como Fórum e Bate-papo, visando a discussão dos assuntos abordados no objeto, e as de publicação e produção dos participantes, como Webfólio e o Diário de Bordo. Além dessas ferramentas, como citado anteriormente, o próprio OA conta com um espaço de inserção de estratégias pedagógicas, visando a construção de um banco de dados dinâmico e a coleta de informações que foram fundamentais para a avaliação do objeto em questão.

Abaixo são apresentados os dados coletados referentes às estratégias pedagógicas voltadas para as interações sociais na EAD e ao uso do objeto. Esses dados subsidiarão o aprimoramento do mesmo, visando a disponibilização de uma versão do $\mathrm{OA}$ mais próxima às necessidades de seus usuários.

Quanto ao auxílio do objeto nos estudos realizados, bem como sua forma de organização e de disponibilização dos materiais, os alunos demonstram que ele contribuiu para a aprendizagem, bem como destacaram a facilidade de navegação e a estruturação do conteúdo. A utilização de um objeto de aprendizagem para a abordagem de um conteúdo foi vista da seguinte forma:

\footnotetext{
${ }^{1}$ Visando a preservação da identidade dos mesmos, a identificação de cada participante será realizada com números de 1 a 30 .
} 
- Gosto muito de ter acesso aos objetos de aprendizagem, pois eles reúnem os conteúdos de maneira organizada e prática para acessarmos e tirarmos dúvidas. (Participante 2)

- Com certeza! Embora ainda devamos estudar mais para saber sobre o conceito e aplicação de objetos de aprendizagem para melhor utilizá-los. (Participante 14)

- Achei a abordagem do conteúdo pontual e objetiva. (Participante 9)

- Muito bem organizado, apresentando temáticas importantes e conceitos relevantes para quem atua na EaD ou tem interesse em atuar. (Participante 14)

- Muito interessante. Tive experiência na elaboração em processos semelhantes, porém em construções extremamente diversas quanto à construção e interpretação dos resultados. (Participante 7 )

- Acredito que o SocioAva reúne de maneira interessante e organizada as informações tratadas ao longo do curso. (Participante 20)

- Bem organizado e norteador, permitindo que o estudante consiga "se encontrar" com facilidade. (Participante 24)

Conforme relatado na apresentação do OA, ele conta com materiais e desafios que visam a problematização da importância das estratégias pedagógicas voltadas para as interações sociais, sendo voltado para a formação continuada dos professores. Sobre essas questões, os participantes verificaram que a falta de uma formação para a atenção a esse aspecto é uma das questões que dificultam o processo. Neste sentido, a formação continuada também foi vista como importante para que essa visão do perfil do aluno da EaD seja considerada nos planejamentos:

- Acredito que o maior desafio está na formação docente, pois muitos estão atuando por ter a formação necessária, mas não estão preparados para utilização das tecnologias, essa parte é uma formação continuada. (Participante 15)

- As habilidades para lidar com as ferramentas e, principalmente, o perfil de quem atua no EaD. Para atuar em EaD precisa ter uma afinidade com as ferramentas, boa vontade e disponibilidade para atender seu aluno. (Participante 19)

- Os docentes devem estimular a participação dos estudantes por meio de recursos atrativos e para isto, deve s aber como utilizar as ferramentas de forma correta, pois o uso não pode ser somente por questões estéticas ou modismos. (Participante 22)

Compreendidos os desafios vistos pelos participantes para o fomento às interações sociais, passou-se para a discussão sobre estratégias pedagógicas específicas que poderiam auxiliar nestas questões. Dessa forma, eles evidenciaram suas concepções sobre a definição de estratégias pedagógicas próprias para esses aspectos na $\mathrm{EaD}$, demonstrando a importância do estudo do conteúdo do objeto, conforme alguns trechos expostos a seguir:

- Certamente o professor deve promover interações, uma vez que a EAD também se sustenta pela troca, pela colaboração, pela discussão e visualização de formas distintas de abordagem entre seus integrantes. Aprendizagem é um processo dinâmico, não estático! (Participante 5)

- É fundamental para se criar um espírito de grupo, ajuda entre os alunas da disciplina. O grau de motivação influencia diretamente para o sucessol resultado do aluno. (Participante 10) 
- Sem duvida deve prover e provocar a interação social entre o grupo. Mas também pode inspirar que os alunos também tenham autonomia para criar essas interações entre o prórpio grupo e entre o professor. (Participante 13)

- Não só pode como deve criar formas de se aproximar dos estudantes, pois é este vinculo que torna a aprendizagem significativa. (Participante 21)

Os participantes foram unanimes em compreender a necessidade de elaboração de estratégias pedagógicas específicas para o fomento das interações sociais. Isto corrobora com a visão apresentada na elaboração do objeto, a respeito da atenção aos aspectos sociais. Tendo em vista que professores iniciantes na modalidade, muitas vezes, não possuem habilidades básicas para esse processo de mediação, promover a reflexão e a atenção a estes aspectos torna-se de extrema importância.

Conforme a compreensão dos participantes, a utilização de OA na prática pedagógica viabiliza aos sujeitos uma participação mais ativa em seu processo de aprendizagem, pois o material oferece diferentes meios pelos quais o aluno poderá construir seu conhecimento. Além disso, por ser disponibilizado em diversas mídias, pode subsidiar os mais diferentes tipos de prática pedagógica, proporcionando espaços de interação e interatividade aos seus usuários. Dessa forma, na produção de um OA é importante que a equipe possua um conhecimento profundo de sua área de atuação, além das diferentes formas de aprendizagem de seus alunos, para que o objeto seja de auxílio a sua prática e consistente com os seus objetivos.

\section{CONSIDERAÇÕES FINAIS}

Entende-se que a utilização de OA na prática pedagógica viabiliza aos sujeitos uma participação mais ativa em seu processo de aprendizagem, pois o material oferece diferentes meios pelos quais o aluno poderá construir seu conhecimento. Além disso, por ser disponibilizado em diversas mídias, pode subsidiar os mais diferentes tipos de prática pedagógica, proporcionando espaços de interação e interatividade aos seus usuários.

O processo de desenvolvimento e de avaliação do SocioAVA_EP demonstrou que promover as interações sociais na Educação a Distância ainda é um desafio, seja para professores iniciantes, ou para os que já atuam na EAD há algum tempo. A forma com que as ferramentas são usadas, os processos de mediação do docente e a distância entre os alunos são algumas das questões que devem ser consideradas na elaboração de estratégias que visem dirimir essas dificuldades. Assim, entende-se que o OA SocioAVA_EP, ao abordar a influência das estratégias pedagógicas com base nos aspectos sociais na educação, focando na educação a distância, pode auxiliar de maneira significativa no aprimoramento da prática pedagógica do professor.

Através da análise dos dados obtidos no estudo de caso pode-se perceber uma grande contribuição do objeto na formação dos professores, sejam reflexões acerca da ressignificação das interações sociais, ou, ainda, uma melhor compreensão do que são os materiais educacionais digitais e objetos de aprendizagem que são estudados ao longo da disciplina.

Nesse sentido, destaca-se a necessidade de considerar os aspectos sociais na educação, em especial, nas práticas realizadas a distância. Embora muito se tenha evoluído em termos tecnológicos nos últimos anos, algumas questões ainda merecem atenção, como a integração dos alunos nos ambientes virtuais. Os AVA utilizados atualmente apresentam diversas ferramentas de interação e dispõem de dados sobre a turma que podem ser de extrema importância para o professor, pois evidenciam o acesso, às formas de interação, o tempo de conexão de seus alunos, entre outras questões. Dessa 
forma, ao acompanhar esses dados, o professor tem a oportunidade de construir, adequar e direcionar suas estratégias de forma a tornar sua prática mais próxima às necessidades de seus alunos.

\section{REFERÊNCIAS}

BEHAR, P.; MACEDO, A. L.; SOUZA, A. P. F. C.; BERNARDI, M. Objetos de Aprendizagem para a Educação a Distância. In: Modelos pedagógicos em educação a distância. Porto Alegre: Artmed, 2009.

BORBA, E. J.; GASPARINI, I. O Uso da Trajetória de Aprendizagem do Aluno em Ambientes Virtuais de Aprendizagem. In: RENOTE - Revista Novas Tecnologias na Educação, v. 13, p. 1-10, 2015.

NESI, T. L.; KALINKE, M. A.; MOTTA, M. S.; MOCROSKY, L. F. Objetos de aprendizagem de matemática: um panorama do que diz em alguns estudos no Brasil. In: RENOTE - Revista Novas Tecnologias na Educação. v. 17, n.1, p. 557-566, 2019.

PIAGET, J. Relações entre a afetividade a inteligência no desenvolvimento mental da criança. Rio de Janeiro: WAK Editora, 2014.

PIAGET, J. Estudos Sociológicos, Rio de Janeiro, Forense, 1973.

SOUZA, A. R. B.; SARTORI, A. S.; ROESLER, J. Mediação pedagógica na educação a distância: entre enunciados teóricos e práticas construídas. Revista Diálogo Educacional, v. 8, n. 24, p. 327-339, 2008.

TORREZZAN, C. A. W.; BEHAR, P. A. Competências para a Construção de Materiais Educacionais Digitais Baseados no Design Pedagógico. In: Patricia Alejandra Behar. (Org.). Competências em Educação a Distância. 1ed.Porto Alegre: Penso, 2014, v. 1, p. 237-262.

TORREZZAN, C. ConstruMed: Metodologia para a construção de materiais educacionais digitais baseados no design pedagógico. 2014. Tese (Doutorado em Informática na Educação), Programa Pós-graduação em Informática na Educação, Centro Interdisciplinar de Novas Tecnologias na Educação, Universidade Federal do Rio Grande do Sul, Porto Alegre, 2014. Disponível em: <http://www.lume.ufrgs.br/handle/10183/106458>. 